\title{
Seguimiento a cinco años en pacientes diabéticos sin síntomas cardíacos estudiados con perfusión miocárdica.
}

\author{
Teresa Massardo ${ }^{1}$, Hernán Prat ${ }^{2}$, Verónica Araya ${ }^{3}$, Isabel Berrocal ${ }^{1^{\star}}$, Rodrigo Jaimovich ${ }^{4}$, \\ René Fernández ${ }^{1}$ \\ 1 Sección Medicina Nuclear del Departamento de Medicina del Hospital Clínico Universidad de Chile \\ 2 Departamento Cardiovascular del Hospital Clínico Universidad de Chile \\ 3 Sección Endocrinología del Departamento de Medicina del Hospital Clínico Universidad de Chile. \\ 4 Medicina Nuclear, Departamento Radiología Pontificia Universidad Católica de Chile. \\ ${ }^{\star}$ Residente CENDEISS (Costa Rica). \\ Financiado por CRP IAEA CHI - 13636
}

Antecedentes: El rendimiento del estudio de perfusión miocárdica en pacientes con Diabetes Mellitus (DM) y sin síntomas coronarios es controvertido.

Objetivo: Efectuar un seguimiento a 5 años para eventos cardíacos mayores en DM asintomáticos estudiados con SPECT miocárdico en esfuerzo.

Método: Se estudiaron 40 pacientes asintomáticos coronarios, con DM conocida por $\geq 5$ años, $>45$ años de edad, con $\geq 2$ factores de riesgo cardiovascular (FRCV) y electrocardiograma basal normal o con alteraciones inespecíficas. Todos efectuaron una prueba de esfuerzo (PE) adecuada y se estudiaron con Sestamibi-Tc $99 \mathrm{~m}$ repetido en 32 casos al $3^{\circ}$ año. Además, se evaluó a 36 controles no diabéticos con $>2$ FRCV. El seguimiento fue de $64 \pm 11$ meses.

Resultados: En el grupo DM, $48 \%$ tenía Hbalc > 7.5\%; en ellos el SPECT mostró isquemia silente (IS) en $30 \%$ y la PE en $18 \%$ del total. No hubo variaciones significativas en SPECT al $3^{\circ}$ año. Hubo una muerte no cardíaca en el $2^{\circ}$ año; en el $4^{\circ}$ año, se produjo una muerte por infarto agudo de miocardio, con mínima isquemia septal y otra asociada a miocardiopatía e insuficiencia cardíaca, con defecto de predominio fijo, ambos con PE negativa. En el grupo control, el $11 \%$ tuvo SPECT anormal y $8 \%$ PE positiva; hubo una muerte no cardíaca. El Odds Ratio para isquemia fue 3.42 [ $\mathrm{IC}=0.99-11.85$ ] entre $\mathrm{DM}$ vs controles y el Hazard Ratio para eventos cardíacos 6.75 [IC $=0.40-108.2]$.

Conclusiones: En DM asintomáticos coronarios el riesgo de IS en SPECT fue mayor que en controles sin DM y con FRCV. Los eventos cardiacos mayores fueron más frecuentes, aunque no significativamente, en DM que en controles. Ello deberá reevaluarse conocidos los resultados del estudio multicéntrico. 


\section{Five year follow-up in Diabetics without coronary artery disease symptoms undergoing Exercise stress and SPECT Myocardial perfusion imaging.}

The clinical value of myocardial perfusion studies in cardiac asymptomatic patients with Diabetes Mellitus (DM) is controversial.

Aim: To conduct a 5 year follow-up study to detect major adverse cardiac events in asymptomatic DM patients using exercise stress and SPECT myocardial perfusion imaging.

Methods: 40 patients with known DM for at least 5 years and without evidence of coronary artery disease, older than 45 years of age with $\geq 2$ cardiovascular risk factors (CVRF) and either normal or minimally altered ECG underwent adequate exercise testing (ET) and 99mTc Sestamibi, repeated 3 years later in 32 cases. We also assessed 36 non-diabetic control patients, with $>2$ CVRF. Mean follow up was $64 \pm 11$ months.

Results: $48 \%$ of DM patients had HbAlc $>7.5 \%$; in them, the SPECT showed silent ischemia (SI) in $30 \%$ and positive ET in $18 \%$. There were no significant variations in SPECT at 3

Introducción: La Diabetes Mellitus (DM) es un factor de riesgo cardiovascular (FRCV) mayor para enfermedad coronaria (EC), condición que tiene gran relevancia e impacto en los costos en salud en diversos niveles de atención. En la actualidad, la muerte de causa cardíaca, tanto por infarto de miocardio (IM) como insuficiencia cardíaca, es predominante en pacientes con DM tipo 2 en los países desarrollados y en algunos en vías de desarrollo. Esta población puede presentar eventos coronarios sin síntomas previos; la isquemia silente (IS) suele estar presente por períodos largos sin ser reconocida y por ende, sin la terapia adecuada. Por otro lado, la prevalencia de IS tiene asociación positiva con el número de FRCV en menores de 75 años. Sobre esta edad habría mayor grado de IS, independiente de la presencia de otros FRCV. ${ }^{1,2}$ Por otra years. There was 1 non-cardiac death in the 2 nd year and 2 deaths, one from acute myocardial infarction associated to minimal septal ischemia and another associated with cardiomyopathy and heart failure, with a fixed perfusion defect. Both patients had a negative ET. In the control group, $11 \%$ had abnormal SPECT and $8 \%$ positive ET and no cardiac deaths were observed. The Odds ratio for ischemia between DM and controls was $3.42[\mathrm{CI}=0.99-11.85]$ and the Hazard Ratio for cardiac events was 6.746 [CI $=0.4205-108.2]$.

Conclusions: In asymptomatic coronary DM patients, the presence of SPECT ischemia detect was higher than in non-DM controls with CVRF. The incidence of major cardiac events was higher, although not significantly, in DM than control patients. This finding should be re-evaluated in the multicenter study.

Key words: Diabetes Mellitus, Silent Ischemia, Myocardial Perfusion, SPECT.

parte, la terapia de IS está en discusión, especialmente en población DM con bajo riesgo de EC considerando, además, que el concepto de DM como un equivalente coronario no está ampliamente aceptado.

La tomografía de fotón único (SPECT) de perfusión miocárdica efectuada con estrés tiene un alto valor diagnóstico y de estratificación de riesgo en pacientes con DM.3,4 Existe controversia sobre la necesidad de realizar tamizaje de rutina mediante técnicas con isquemia inducida a pacientes asintomáticos cardíacos con $\mathrm{DM}^{3,5,6}$ Se debe considerar que el tipo de prueba de provocación utilizada tiene valor pronóstico en sí misma, debido a que pacientes con DM referidos a estrés farmacológico tienen mayor gravedad y hasta 7 veces mayor mortalidad comparados con los que pueden realizar ejercicio ${ }^{7,8}$ 
El estudio DIAD realizado en EEUU demostró que no era aconsejable efectuar tamizaje en población DM asintomática evaluando a grupos sometidos o no a SPECT de perfusión miocárdica con un estresor farmacológico, la adenosina (con seguimiento promedio de $4.8 \pm 0.9$ años) porque se encontró baja tasa acumulada de eventos coronarios de $2.9 \%$ y alrededor de $0.6 \%$ de eventos anuales, sin diferencia significativa entre los grupos estudiados. Los pacientes con SPECT normal o con defecto pequeño tuvieron menor tasa de eventos coronarios que aquellos con defectos moderados o grandes $(0.4 \%$ versus $2.4 \%$ al año; $\mathrm{p}=0.001)$. El valor predictivo positivo de los defectos de perfusión moderados o grandes fue $12 \%$. La revascularización coronaria fue baja en los grupos estudiados y no estudiados con SPECT (5.5\% y 7.8\%, p=ns). En el seguimiento a 5 años la tasa anual global de eventos coronarios fue de menos de $1 \%$ salvo en los pacientes con mayor riesgo cardiovascular que correspondió a 2\%,5,9-

${ }^{11} \mathrm{La}$ IS en pacientes DM asintomáticos coronarios tiene prevalencia variable en algunas series aunque no es bien conocida en todas las zonas geográficas. En un trabajo retrospectivo reciente de IS utilizando diversos tipos de estrés, la prevalencia de IS en el subgrupo con DM y 2 o más FRCV fue de $13 \%$ vs $6 \%$, comparado con subgrupo no diabéticos $(\mathrm{p}=0.001) \mathrm{y}$, además, fue más severa $(\mathrm{p}=0.04) .^{10}$

En otro análisis multivariado de DM asintomáticos versus controles con SPECT en estrés normal, la fracción de eyección ventricular izquierda (FEVI) post estrés y presencia de DM fueron predictores independientes de muerte por causa cardíaca o IM no fatal. ${ }^{12}$ Sin embargo, ese estudio, denominado IDIS, incluía sujetos infartados y pacientes con insuficiencia cardíaca, y posiblemente EC conocida, y los pacientes presentaban mayor riesgo coronario que en el estudio DIAD. ${ }^{13}$

En este contexto, el Organismo Internacional de Energía Atómica (IAEA) ha llevado a cabo un proyecto multicéntrico en 12 países en vías de desarrollo, incluyendo a Chile, con 392 pacientes DM tipo 2 asintomáticos coronarios observándose que la IS está presente en ellos en un $26 \%$ vs. $14 \%$ en controles no diabéticos ( $\mathrm{p}<0.001)$; el estudio evaluó la perfusión miocárdica isotópicamente y mediante prueba de esfuerzo (PE) en todos ellos. ${ }^{14}$ Se desconoce mayormente la prevalencia de los eventos coronarios en presencia de IS en pacientes con DM y FRCV con capacidad de efectuar una adecuada PE. Por esta razón, el objetivo de nuestro trabajo fue realizar un seguimiento a 5 años en un grupo de pacientes chilenos con DM y asintomáticos coronarios estudiados con SPECT de perfusión miocárdica en esfuerzo, con el fin de pesquisar eventos cardíacos mayores en la evolución alejada.

Material y métodos: Este es un estudio prospectivo multinacional del que presentamos los casos chilenos, efectuado en pacientes voluntarios que no estaban en control cardiológico y eran asintomáticos cardíacos. Los pacientes eran provenientes de consulta endocrinológica o general y algunos sin control médico habitual. Se obtuvo consentimiento informado al ingreso a protocolo y el estudio fue aprobado por el Comité de Ética Científico del Hospital Clínico de la Universidad de Chile.

Sujetos: Se evaluó a 40 pacientes sin síntomas de enfermedad coronaria, con DM tipo 2 conocida por 5 años o más, mayores de 45 años de edad; con otros FRCV distintos de su DM (hipertensión arterial, tabaquismo, dislipidemia, antecedentes familiares directos de EC) y electrocardiograma normal o cercano a lo normal, aceptándose alteraciones de repolarización, hipertrofia ventricular y bloqueo completo de rama derecha. Además, se evaluó a 36 controles con más de un FRCV, no diabéticos y también asintomáticos coronarios.

Como criterios de exclusión estaban la EC conocida, accidente vascular encefálico, incapacidad de efectuar test de esfuerzo, alteraciones electrocardiográficas en reposo como arritmias, bloqueo completo de rama izquierda o presencia de onda $\mathrm{Q} u$ otra sospecha de EC. No se incluyeron pacientes con daño renal conocido.

Técnica: Todos los pacientes efectuaron prueba de esfuerzo electrocardiográfica (PE) con Treadmill General Electric CASE-1, obteniendo más de $85 \%$ de frecuencia cardíaca máxima teórica, con supervisión cardiológica y registro de 12 derivaciones, inyectando el radiofármaco I.V., en máximo estrés.

El estudio tomográfico SPECT con Sestamibi-Tc99m se realizó en gamma cámara SPECT doble cabezal Siemens. Se utilizó entre 12 y $30 \mathrm{mCi}$ (444 y $1.110 \mathrm{MBq}$ ) por inyección en protocolo de 1 día y entre 20-25 mCi (740$925 \mathrm{MBq}$ ) en 2 días. Se adquirió en fase de post-esfuerzo y reposo con protocolo de 1 o 2 días; todos los estudios incluían gatillado electrocardiográfico con 8 "frames" en ambas fases. Se usó procesamiento iterativo y corrección de movimiento en caso necesario. El análisis de SPECT 
fue de tipo visual de cortes tomográficos reorientados y en modo cine. La cuantificación utilizó software Cedars® QGS y QPS para evaluar función y perfusión.

Seguimiento: Se siguió a todos los pacientes mediante contacto telefónico y análisis de ficha clínica, salvo un caso en que no fue posible contactar por ningún medio; El tiempo promedio fue $64 \pm 11$ meses (rango: 10-87). El estado de supervivencia se verificó mediante datos del Registro Civil. Se analizó perfil lipídico, creatininemia, glicemia en ayunas, hemoglobina glicosilada en DM y microalbuminuria y Proteína $\mathrm{C}$ reactiva ultrasensible (PCR us) en los DM al tercer año.

Se realizó un nuevo estudio de perfusión en 32 pacientes con DM al $3^{\circ}$ año.

No hubo intervención farmacológica dirigida, y los tratantes, si los había, recibieron el resultado de los SPECT. Los casos que no tenían un médico a cargo fueron aconsejados para que consultaran, especialmente, en los casos positivos.

Análisis Estadístico: se usaron pruebas de $\mathrm{t}$ de Student para comparar promedios con $p$ significativa $<0.05$, prueba de Fisher para muestra pequeñas y análisis porcentual de sobrevida con log rank (Software Prism ${ }^{\circledR}$ ).

Resultados: Algunas características demográficas y del perfil lipídico de ambos pacientes y controles se muestran en la Tabla 1. El 48\% de los pacientes con DM tuvo valores de $\mathrm{HbA} 1 \mathrm{c}>7.5 \%$ lo que no varió significativamente a los 3 años. La microalbuminuria aislada medida al control del tercer año fue $77 \pm 195 \mathrm{mg} / \mathrm{L}$. Salvo en la glicemia, no hubo diferencias entre DM y controles en sus FR (Tabla 2).

\begin{tabular}{|l|l|l|l|}
\begin{tabular}{|l|l|} 
Tabla 1. Datos demográficos y de laboratorio basales \\
de paciéntes con DM y controles al ingreso a protocolo.
\end{tabular} \\
\hline & DM & Controles & p \\
\hline Edad (años) & $59 \pm 8$ & $57.1 \pm 11$ & ns \\
\hline Sexo masculino (\%) & 60 & 64 & ns \\
\hline IMC (kg/m2) & 27.8 & 28.2 & ns \\
\hline Glicemia ayunas & $152 \pm 58$ & $93.3 \pm 12$ & $<0.0001$ \\
\hline HbAlc (\%) & $8.3 \pm 2.2$ & - & - \\
\hline Creatininemia & $0.91 \pm 0.2$ & $0.90 \pm 0.2$ & ns \\
\hline Colesterol total & $189 \pm 33$ & $198 \pm 32$ & ns \\
\hline HDL & $45 \pm 11$ & $48 \pm 12$ & ns \\
\hline LDL & $112 \pm 31$ & $112 \pm 34$ & ns \\
\hline Triglicéridos & $170 \pm 79$ & $156 \pm 67$ & ns \\
\hline
\end{tabular}

\begin{tabular}{|c|c|c|c|}
\hline FRCV & DM & Controles & $\mathrm{p}$ \\
\hline Tabaco & $57.5 \%$ & $58.3 \%$ & ns \\
\hline Hipertensión arterial & $65 \%$ & $75 \%$ & ns \\
\hline Dislipidemia & $72.5 \%$ & $80.5 \%$ & $\mathrm{~ns}$ \\
\hline Antecedentes familiares de EC & $37.5 \%$ & $61.1 \%$ & ns \\
\hline Número FRCV (distintos que DM) & $2.3 \pm 1.1$ & $2.8 \pm 0.9$ & ns \\
\hline
\end{tabular}

En los DM, el SPECT mostró IS en 12/40 casos (30\%) y la PE fue positiva en $3 / 40$ (18\%) del total, lo que fue significativamente diferente de los controles $(\mathrm{p}=0.0198)$ Los defectos isquémicos fueron leves o moderados y sólo en un caso la IS fue severa (Figura 1). Un caso presentaba defecto fijo que correspondía a infarto miocárdico no pesquisado al ECG. $\mathrm{Al} 3^{\circ}$ año, hubo solo leves cambios en menor reversibilidad y aumento de tamaño del defecto fijo (Figura 2).

El rango de la FEVI post-estrés fue 33-75\% para los DM y 35-75\% para los controles al inicio del protocolo $(\mathrm{p}=\mathrm{ns})$, sin cambios a los 3 años en el grupo DM ( $\mathrm{p}=\mathrm{ns})$. Inicialmente los volúmenes de fin de diástole (VFD) post estrés variaron entre 29-150 ml para los DM y 32-116 ml para los controles (Tabla 3). Tampoco hubo cambios a los 3 años en los DM.

\begin{tabular}{|l|l|l|l|}
\hline \begin{tabular}{|l|l|} 
Tabla 3. Datos de la PE electrocardiográfica iniciales de los DM y \\
controles y del estudio funcional SPECT postestrés inicial.
\end{tabular} \\
\hline & DM & Controles & p \\
\hline METs & $8.9 \pm 2.3$ & $9.6 \pm 2.2$ & ns \\
\hline FCMT (\%) & $98.7 \pm 6$ & $96.1 \pm 5$ & ns \\
\hline Duración PE (min) & $7.0 \pm 2.2$ & $7.3 \pm 2$ & ns \\
\hline FEVI postestrés (\%) & $68 \pm 10$ & $69 \pm 8$ & ns \\
\hline VFD postestrés (ml) & $62 \pm 22$ & $66 \pm 19$ & ns \\
\hline
\end{tabular}

(MET) Equivalente metabólico. (PE): Prueba de esfuerzo. (FCMT): Frecuencia cardíaca máxima teórica. (FEVI): Fracción de eyección del ventrículo izquierdo. (VFD): Volumen de fin de diástole del ventrículo izquierdo.

En el tercer año, hubo microalbuminuria ( $>30 \mathrm{mg} / \mathrm{L}$ ) en $31 \%$ de los DM evaluados. El $44 \%$ de los pacientes con microalbuminuria alterada tenían IS. La PCR us estuvo alterada en $13 \%$ de los DM y solo 1 de esos casos presentó isquemia.

En el seguimiento, efectuado en los DM hubo 3 muertes, todas en pacientes de sexo masculino, la primera durante el $2^{\circ}$ año por complicaciones de VIH. Las otras 2 muertes, ocurrieron durante el $4^{\circ}$ año, una por infarto agudo 

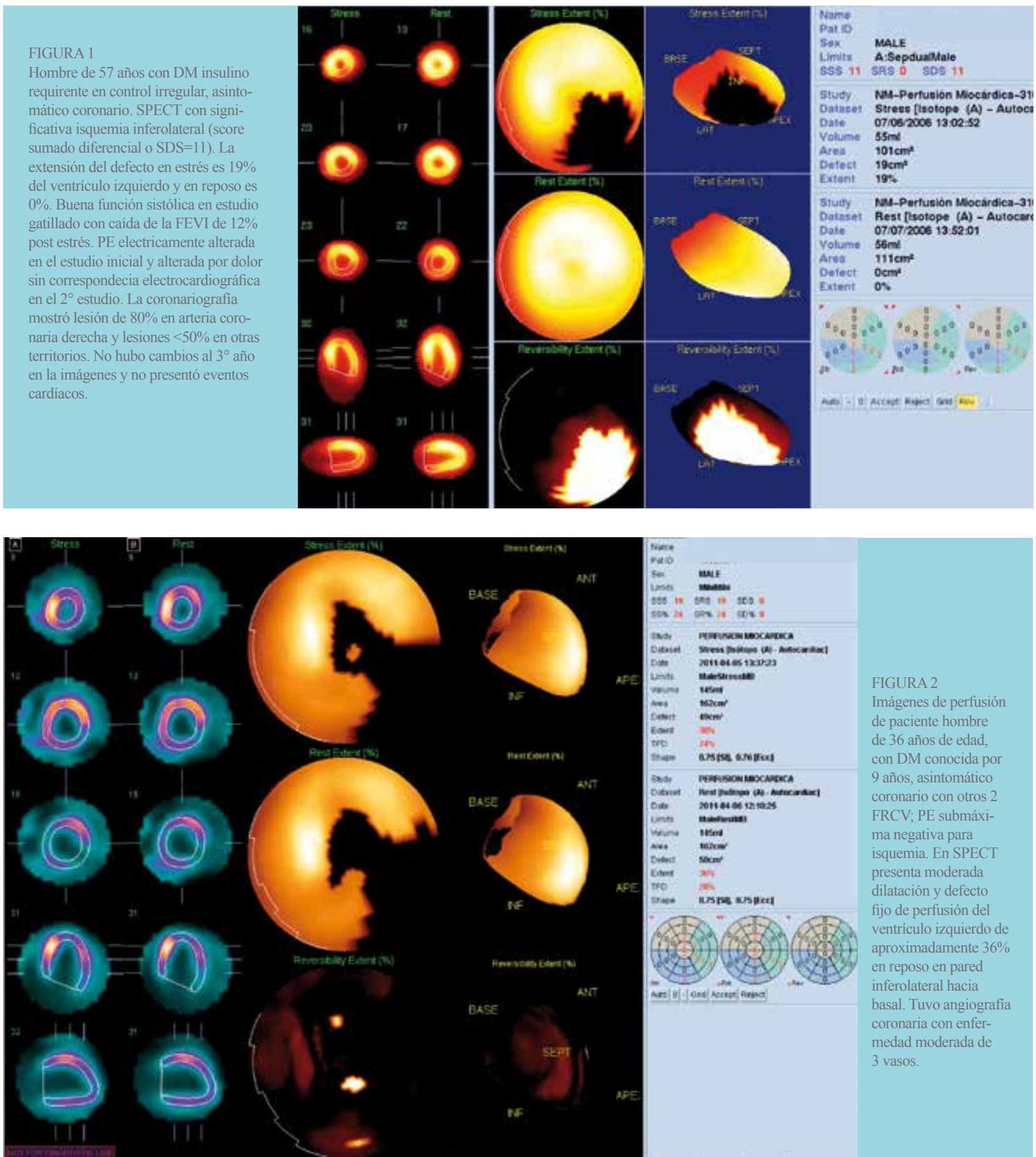

de miocardio en paciente hospitalizado que correspondió a sujeto de 68 años, con DM conocida 8 años antes del ingreso a protocolo, cuyos SPECT mostraban mínima isquemia septal; el otro deceso también ocurrió en paciente de 36 años, con miocardiopatía e insuficiencia cardíaca.
Debido a disnea de 6 meses de evolución y presencia en SPECT de dilatación con función sistólica global disminuida y defecto de perfusión de predominio fijo (Figuras 2 y 3$)$ se realizó angiografía al $2^{\circ}$ año que mostró EC moderada de 3 vasos y arterias de mal desarrollo. Estos últimos 


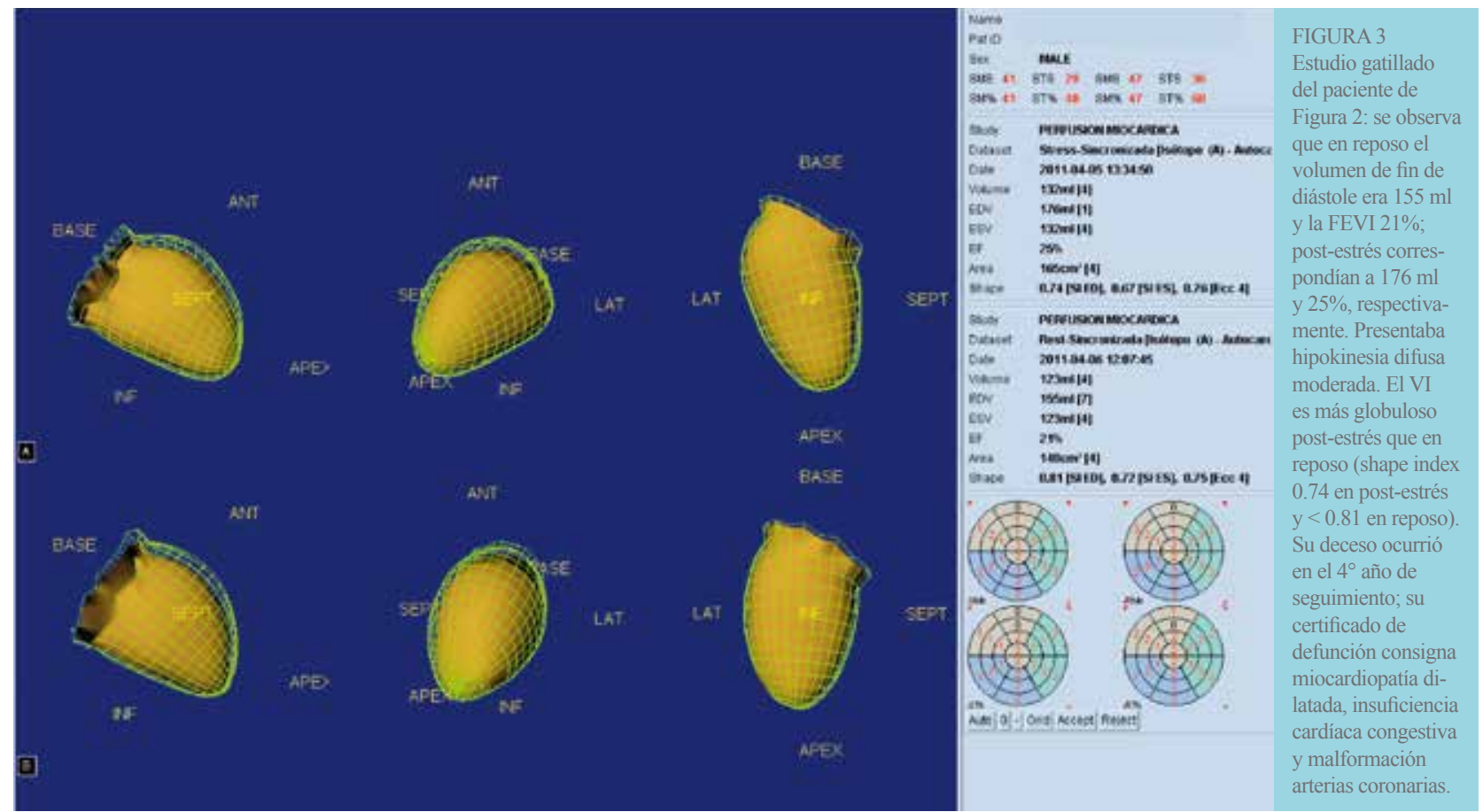

dos casos tuvieron PE negativa y sus estudios SPECT al $3^{\circ}$ año tampoco presentaban cambios significativos.

No hubo cambios significativos al comparar los valores sanguíneos iniciales del grupo DM comparados con los medidos al $3^{\circ}$ año. Tampoco hubo cambios en los parámetros de la PE y de función ventricular al $3^{\circ}$ año en los pacientes con DM evaluados. El Odds Ratio de presentar isquemia en el SPECT con ejercicio fue 3.42 [Intervalo de confianza (IC): 0.99-11.85] en los DM versus los controles. Hubo discordancia entre la positividad de los resultados de SPECT y PE en varios casos.

El $32.5 \%$ de los DM y el $58.3 \%$ de los controles con FRCV logró $\geq 10$ METS inicialmente en la PE ( $\mathrm{p}=0.037$ ). En los controles, $4 / 36$ pacientes $(11 \%)$ tuvieron SPECT de perfusión anormal y la PE fue positiva en 3/36 (8\%). En este grupo sólo se observó una muerte no cardíaca durante el $1^{\circ}$ año por melanoma maligno. No hubo diferencias significativas en la sobrevida sin eventos cardíacos mayores entre DM y controles. El Hazard Ratio de presentar eventos cardíacos mayores entre DM y controles fue 6.746, aunque el IC fue muy amplio (0.4205-108.2)

Discusión: Los resultados de este estudio muestran que el SPECT fue superior a la PE para detectar isquemia en pacientes con DM sin evidencia de enfermedad coro- naria, datos concordantes con lo encontrado en el grupo multicéntrico del cual este estudio forma parte. ${ }^{14}$ Además, los 2 casos con eventos cardíacos tuvieron SPECT positivo con PE negativas que alcanzaron 7 METS en el caso con infarto agudo y 10 METS en el paciente con insuficiencia cardíaca. El seguimiento a 5 años del subgrupo chileno de DM asintomáticos coronarios demostró una tasa de eventos de $5 \%$ que, aunque baja, no es despreciable al contrastarla con la ausencia de eventos a 5 años, en el grupo control. Este valor aparece mayor que en estudio DIAD, pero el número reducido de pacientes limita el significado de nuestros hallazgos.

En un análisis retrospectivo de estratificación de riesgo de pacientes de EE.UU con y sin DM usando SPECT de perfusión en esfuerzo o estrés farmacológico, la tasa anual de eventos cardíacos fue significativamente menor para los DM sometidos a ejercicio que a adenosina. Los pacientes DM sin EC conocida también tuvieron menor tasa de eventos que el resto de los pacientes DM. Comparando solo los casos con perfusión anormal, la tasa anual de eventos entre los sometidos a ejercicio no fue distinta entre DM y no DM en un seguimiento promedio de $2.4 \pm 1.4$ años. ${ }^{6}$

Un grupo de investigadores de otra región con alta prevalencia de DM, presentó recientemente una incidencia 
de eventos cardíacos mayores de $18.3 \%$ en pacientes DM con EC conocida o sospechada, versus $9 \%$ en no DM $(\mathrm{p}<0.001)$, lo que se asemeja a nuestros hallazgos preliminares. Ellos realizaron SPECT con adenosina a 300 pacientes con seguimiento promedio de $26.7 \pm 8.8$ meses. La perfusión anormal en estrés fue un predictor independiente y potente para los eventos mayores con valor pronóstico incremental en la estratificación de riesgo cardiovascular de DM. ${ }^{15}$ Por otra parte, el resultado del SPECT en 1.427 DM asintomáticos estratificados de acuerdo al resultado del SPECT en bajo, mediano y alto riesgo, mostró diferencias significativas en la tasa de mortalidad anual entre los grupos. ${ }^{16}$

Otro trabajo prospectivo reciente presenta 575 pacientes consecutivos con DM con riesgo intermedio de EC, $40.3 \%$ de ellos con EC conocida y $29 \%$ asintomáticos. Tuvieron isquemia significativa (definida como $\geq 10 \%$ del ventrículo izquierdo) $21.9 \%$. Durante el seguimiento con mediana de 4.4 años, la tasa de eventos cardíacos mayores/fatales fue moderada, 2.6\%/año (muerte car-

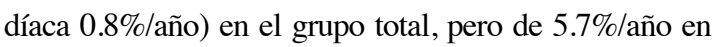
los con isquemia ( $\mathrm{p}<0.001)$. La tasa de eventos aumentó después de los 2 años. Fueron predictores independientes de muerte cardíaca o IM, la EC conocida, la isquemia observada con estrés farmacológico, y la isquemia con SPECT. Alrededor de $1 / 3$ de los pacientes con eventos tenían SPECT normal, lo que para los autores indica que se requiere mejorar la estratificación previo al estudio con SPECT. ${ }^{17}$ Esto último, diferencia ese trabajo del nuestro en el que no se había solicitado el examen como parte de su manejo habitual.

De acuerdo al estudio DIAD en DM asintomáticos cardíacos, el uso de estudios con provocación de isquemia no se aconseja entre otras causas por la baja capacidad de detección de la prueba, número de eventos relativamente bajo que no parecen afectar la evolución y además su alto costo. Por ello, las recomendaciones actuales para estudios de provocación en DM asintomáticos $\mathrm{CV}$, desaconsejan su uso rutinario ${ }^{18}$. Sin embargo, la población en que fue efectuado el estudio DIAD, el pronóstico era favorable con seguimiento estrecho, terapia adecuada y evaluación de aparición de síntomas isquémicos. Nosotros consideramos que en DM asintomáticos el número de FRCV clásicos y otros recientemente conocidos como cocaína, insuficiencia renal, PCR alterada, la capacidad de ejercicio y la edad, debieran ser parámetros que individualmente justifiquen los estudios de perfusión con provocación de isquemia. También debe recordarse que la PE entrega información referente a la capacidad funcional del paciente que no se obtiene de los estudios con vasodilatadores. El obtener sobre 10 METS en la PE es buen predictor de ausencia de eventos coronarios en el seguimiento, independiente de otros FRCV e incluso de la presencia de isquemia. ${ }^{21,22}$

Respecto a la asociación de IS y presencia de microalbuminuria, también la observamos (en 44\%) como otros autores ${ }^{20}$, para quienes aquella fue el único predictor de IS en DM tipo 2. En este estudio sólo el paciente con insuficiencia cardíaca, de los con evento mayor, la presentó. Entre las fortalezas del trabajo presentado consideramos su carácter prospectivo en pacientes con DM de más de 5 años de evolución, que tanto el grupo de DM como el control, son asintomáticos coronarios con más de 1 FRVC, que todos fueron evaluados con perfusión miocárdica mediante $\mathrm{PE}$ adecuada con un seguimiento relativamente largo. Entre sus debilidades, la principal está el incluir un número reducido de pacientes nacionales y no contar con datos clínicos de certeza respecto a la terapia médica recibida.

\section{Conclusión}

En una muestra pequeña de pacientes asintomáticos coronarios chilenos con FRCV, el riesgo de IS es mayor en portadores de DM que en controles no DM. El trabajo confirma que el estudio SPECT permite efectuar estratificación de riesgo seleccionando los pacientes asintomáticos isquémicos. Estos datos deben ser re-evaluados en el estudio multicéntrico. 


\section{Referencias:}

1. MALHOTRA S, SHARMA R, KLINER DE, FOLLANSBEE WP, SOMAN P. Relationship between silent myocardial ischemia and coronary artery disease risk factors. $\mathbf{J}$ Nucl Cardiol. 2013; 20: 731-8.

2. PETRETTA M, FIUMARA G, PETRETTA MP, CUOCOLO A. Detection of silent myocardial ischemia: is it clinically relevant?. J Nucl Cardiol. 2013; 20: 707-10.

3. HELLER GV. Evaluation of the patient with diabetes mellitus and suspected coronary artery disease. Am J Med. 2005; 118: 9S-14S.

4. BAX JJ, YOUNG LH, FRYE RL, BONOW RO, STEINBERG HO, BARRETT EJ. American Diabetes Association. Screening for coronary artery disease in patients with diabetes. Diabetes Care. 2007; 30: 2729-2736.

5. ISKANDRIAN AE, HAGE FG. Declining frequency of ischemia detection using stress myocardial perfusion imaging. J Am Cardiol. 2013; 61: 1066-8.

6. GHATAK A, PADALA S, KATTEN DM, POLK DM, HELLER GV. Risk stratification among diabetic patients undergoing stress myocardial perfusion imaging. J Nucl Cardiol. 2013; 20: 529-38

7. ALBERS AR, KRICHAVSKY MZ, BALADY GJ. Stress Testing in Patients With Diabetes Mellitus. Diagnostic and Prognostic Value. Circulation. 2006; 113: 583-592.

8. VANZETTO G, HALIMI S, HAMMOUD T, FAGRET D, BENHAMOU PY, CORDONNIER D, et al. Prediction of cardiovascular events in clinically selected high-risk NIDDM patients. Prognostic value of exercise stress test and thallium-201 single-photon emission computed tomography. Diabetes Care. 1999; 22: 19-26.

9. WACKERS FJ, YOUNG LH, INZUCCHI SE, CHYUN DA, DAVEY JA, BARRETT EJ, et al. Detection of silent myocardial ischemia in asymptomatic diabetic subjects. The DIAD study: A randomized controlled Trial. Diabetes Care. 2004; 27: 1954-61.

10. YOUNG LH, WACKERS FJ, CHYUN DA, DAVEY JA, BARRETT EJ, TAILLEFER R, et al; DIAD Investigators. Cardiac outcomes after screening for asymptomatic coronary artery disease in patients with type 2 diabetes: the DIAD study: a randomized controlled trial. JAMA. 2009; 301: 1547-55.

11. BANSAL S, WACKERS FJ, INZUCCHI SE, CHYUN DA, DAVEY JA, STAIB LH, et al; DIAD study investigators. Five-year outcomes in high-risk participants in the Detection of Ischemia in Asymptomatic Diabetics (DIAD) study: a post hoc analysis. Diabetes Care. 2011; 34: 204-9.

12. ACAMPA W, PETRETTA M, EVANGELISTA L, DANIELE S, XHOXHI E, DE RIMINI ML, et al. Myocardial perfusion imaging and risk classification for coronary heart disease in diabetic patients. The IDIS study: a prospective, multicentre trial. Eur J Nucl Med Mol Imaging. 2012; 39: 387-95.

13. FLOTATS A. Myocardial perfusion imaging: a plus for coronary risk classification in diabetics. Eur J Nucl Med Mol Imaging. 2012; 39: 383-6.

14. HAGE FG, LUSA L, DONDI M, GIUBBINI R, ISKANDRIAN AE. IAEA Diabetes Investigators. Exercise stress tests for detecting myocardial ischemia in asymptomatic patients with diabetes mellitus. Am J Cardiol. 2013; 112: 14-20.

15. KASIM M, CURRIE GM, TJAHJONO M, SISWANTO BB, HARIMURTI GM, KIAT H. Myocardial Perfusion SPECT utility in predicting cardiovascular events among indonesian diabetic patients. Open Cardiovasc Med J. 2013; 7: 82-9.

16. RAJAGOPALAN N, MILLER TD, HODGE DO, FRYE RL, GIBBONS RJ. Identifying high-risk asymptomatic 
diabetic patients who are candidates for screening stress single-photon emission computed tomography imaging. J Am Coll Cardiol. 2005; 45: 43-49.

17. BOURQUE JM, PATEL CA, ALI MM, PEREZ M, WATSON DD, BELLER GA. Prevalence and predictors of ischemia and outcomes in outpatients with diabetes mellitus referred for single-photon emission computed tomography myocardial perfusion imaging. Circ Cardiovasc Imaging. 2013; 6: 466-77.

18. HENDEL RC, BERMAN DS, DI CARLI MF, HEIDENREICH PA, HENKIN RE, PELLIKKA PA, et al. ACCF/ ASNC/ACR/ AHA/ASE/SCCT/SCMR/SNM. 2009 appropriate use criteria for cardiac radionuclide imaging: a report of the American College of Cardiology Foundation Appropriate Use Criteria Task Force, the American Society of Nuclear Cardiology, the American College of Radiology, the American Heart Association, the American Society of Echocardiography, the Society of Cardiovascular Computed Tomography, the Society for Cardiovascular Magnetic Resonance, and the Society of Nuclear Medicine. J Am Coll Cardiol. 2009; 53: 2201-29.
19. MASSARDO T, BERROCAL I, CASTRO G, MUÑOZ MP, SANTIS N, PADILLA P, et al. Valor del índice de excentricidad del ventrículo izquierdo con tomografía SPECT de perfusión miocárdica en diversas condiciones. Rev Chil Cardiol 2011; 31: 132 -139.

20. GIOVACCHINI G, CAPPAGLI M, CARRO S, BORRINI S, MONTEPAGANI A, LEONCINI R, et al. Microalbuminuria predicts silent myocardial ischaemia in type 2 diabetes patients. Eur J Nucl Med Mol Imaging. 2013;40:548-57

21. BOURQUE JM, HOLLAND BH, WATSON DD, BELLER GA. Achieving an exercise workload of $>$ or $=10$ metabolic equivalents predicts a very low risk of inducible ischemia: does myocardial perfusion imaging have a role? J Am Coll Cardiol. 2009 4;54:538-45

22. BOURQUE JM, CHARLTON GT, HOLLAND BH, BELYEA CM, WATSON DD, BELLER GA. Prognosis in patients achieving $\geq 10$ METS on exercise stress testing: was SPECT imaging useful? J Nucl Cardiol. 2011;18:230-7 